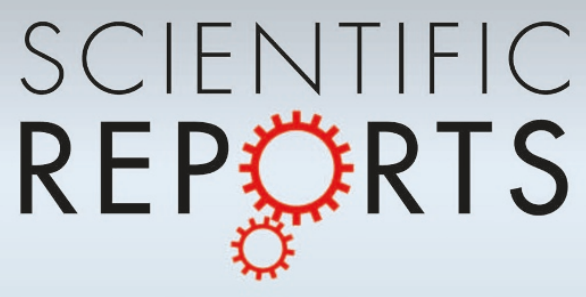

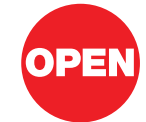

SUBJECT AREAS:

SYNTHESIS

ELECTRONIC MATERIALS AND

DEVICES

CARBON NANOTUBES AND

FULLERENES

MOLECULAR ENGINEERING

Received

27 March 2012

Accepted

3 September 2012

Published

17 September 2012

Correspondence and requests for materials should be addressed to Y.-Q.L. (liuyq@iccas. ac.cn)

\section{Production of Graphite Chloride and Bromide Using Microwave Sparks}

\author{
Jian Zheng, Hong-Tao Liu, Bin Wu, Chong-An Di, Yun-Long Guo, Ti Wu, Gui Yu, Yun-Qi Liu \& Dao-Ben Zhu
}

Key Laboratory of Organic Solids, Beijing National Laboratory for Molecular Sciences, Institute of Chemistry, Chinese Academy of Sciences, Beijing 100190, PR China.

Chemically modified graphite is an economical material with promising applications in its own right or as an intermediate in the synthesis of graphene. However, because of its extreme chemical inertness, to date only two methods-oxidation and fluorination-have been found which can modify graphite with high yield and large throughput. Herein, we describe a third chemical approach for the synthesis of large quantities of highly modified graphite which uses a microwave-sparks-assisted halogenation reaction. The resulting graphite halide can easily be exfoliated into monolayer graphene in organic solvents. The structure and electronic properties of the original graphene can be recovered after thermal annealing of the graphene halide. Furthermore, the graphene halide can be further modified by a variety of organic functional groups. Solution-processed field-effect transistors based on the graphene halides resulted in device performances were comparable to, or even better than, that of graphene oxide.

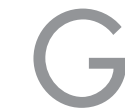
raphene has attracted much attention in the last few years because of its outstanding electronic, mechanical, and thermal properties ${ }^{1-4}$. The fascinating properties of graphene make it suitable for applications in many fields such as energy storage materials, polymer composites, and mechanical resonators ${ }^{4-14}$. Early studies mainly focused on the production of high-quality pristine graphene, investigation of its unique physical properties, and fabrication of prototype devices. It is generally believed that chemical functionalization of graphene will open up a new pathway for an almost unlimited number of graphene-related materials, and provide materials with unprecedented versatility for a range of applications, by taking advantage of their larger bandgap, better solubility in large-scale solution processes and new functionalities. However, much less is known about the chemical functionalization of pristine graphene, mainly due to the fact that it is much more chemically inert than carbon nanotubes and fullerenes ${ }^{15}$; this has posed a formidable experimental challenge, significantly limiting the further development of graphene chemistry.

Chemically modified graphite is an economical material with promising applications in its own right or as an intermediate in the synthesis of graphene. If modified graphene could be obtained from graphite in large quantities it would be an attractive material for use in many composite materials or solution applications. However, graphite is one of the most chemically inert materials known. Although several groups have succeeded in modifying monolayer graphene with a low degree of conversion ${ }^{16,17}$, to date only two methods-oxidation and fluorination-have been successfully employed to modify graphite in high yield ${ }^{18-26}$. But these two methods both suffer from their own drawbacks. The functional groups on graphene oxide are a mixture of different types, which are hard to substitute or fully remove from the surface of graphene, whilst the fluorination reaction relies on special equipment available in a very limited number of laboratories because of the high toxicity and corrosive nature of fluorine. Therefore developing a simple, reliable and effective methodology for the functionalization of graphene, which can be carried out in ordinary laboratories, remains a considerable challenge.

It is generally believed that, unlike fluorine, other halogens cannot react directly with graphite. Recently, Dai and Liu's groups reported the chlorination of monolayer graphene by plasma and photochemical methods, respectively ${ }^{27,28}$. However there has still been no report of the successful high degree chlorination of graphite, although some graphite materials with either very low $\mathrm{Cl}$ or $\mathrm{Br}$ content or modified only on the surface have been obtained $^{29-31}$. Here, we report a microwave-spark (MiW-S) assisted reaction that allows the direct production of $\mathrm{Cl}$ - and $\mathrm{Br}$-functionalized monolayer graphene sheets from graphite with high efficiency for the first time. The $\mathrm{Cl}$ and $\mathrm{Br}$ atoms are covalently functionalized on the graphene sheets with a high $\mathrm{Cl}$ or $\mathrm{Br}$ atom percentage, up to $21 \%$ and $4 \%$ ( $44 \%$ and $21 \%$ by weight), respectively. 


\section{Results}

In a typical experiment (Figure 1a), expandable graphite and either liquid chlorine or bromine were added to a flask which was immersed in liquid nitrogen. The flask was irradiated in a microwave oven (700 W, $2450 \mathrm{MHz}$ ) for $5 \mathrm{~s}$. Graphite displays strong microwave absorption because of its low resistance, with a dramatic temperature increase (to $800-1500^{\circ} \mathrm{C}$, as measured by an infrared thermometer) accompanied by luminous sparks ${ }^{32-34}$. At such high temperatures the graphite expands to $\sim 200$ times its original volume and emits large quantities of hot graphite flakes which can react directly with the halogen. The resulting hot graphite halide became coated on the inside surface of the flask (Figure 1b) thus being immediately quenched to very low temperature, and avoiding thermal decomposition (Figure S1). It has been reported that some materials can be ionized to a plasma under MiW irradiation ${ }^{35}$. Therefore, we suggest that the halogen may become slightly ionized under MiW irradiation in our experiment, and that this may also favor the halogenation reaction. After the reaction, the sample was annealed at $150^{\circ} \mathrm{C}$ to evaporate the adsorbed halogen under a reduced pressure of $\sim 10^{-3}$ mbar. The MiW-S method is very different from the ordinary microwave procedure which mostly relies on excitement of molecular vibrations. If the graphite was first dispersed into small sheets, which reduced the amount of sparks formed under subsequent $\mathrm{MiW}$ irradiation, the halogen content was reduced to less than $0.5 \%$, showing that the sparks play a key role in the reaction. For the chlorination reaction, the liquid state of $\mathrm{Cl}_{2}$ was more efficient than the gaseous state of $\mathrm{Cl}_{2}$ - which only afforded a low $\mathrm{Cl}$ atom content of less than $2 \%$. This is because the liquid $\mathrm{Cl}_{2}$ can effectively intercalate between the graphite layers, efficiently react with the graphite sheets and help to rapidly cool the hot graphite chloride product. Our method results in a $\mathrm{G}-\mathrm{Cl}$ yield of $\sim 10 \%$, which could potentially be improved to $50-80 \%$ with further processing.

In contrast to hydrophilic graphene oxide (GO), the resulting graphite halide cannot be dispersed in water. However, it was easily dispersed in organic solvents, such as dimethylformamide (Figure 1c and $1 \mathrm{~d}$ ) or dioxane, and became exfoliated into graphene chloride $(\mathrm{G}-\mathrm{Cl})$ or graphene bromide $(\mathrm{G}-\mathrm{Br})$. The concentration of $\mathrm{G}-\mathrm{Cl}$ was as high as $0.5 \mathrm{mg} / \mathrm{mL}$, and it was stable against reaggregation, demonstrating its capability for use in solution-processing methods. The concentration of $\mathrm{G}-\mathrm{Br}$ was lower than that of $\mathrm{G}-\mathrm{Cl}$, which can be

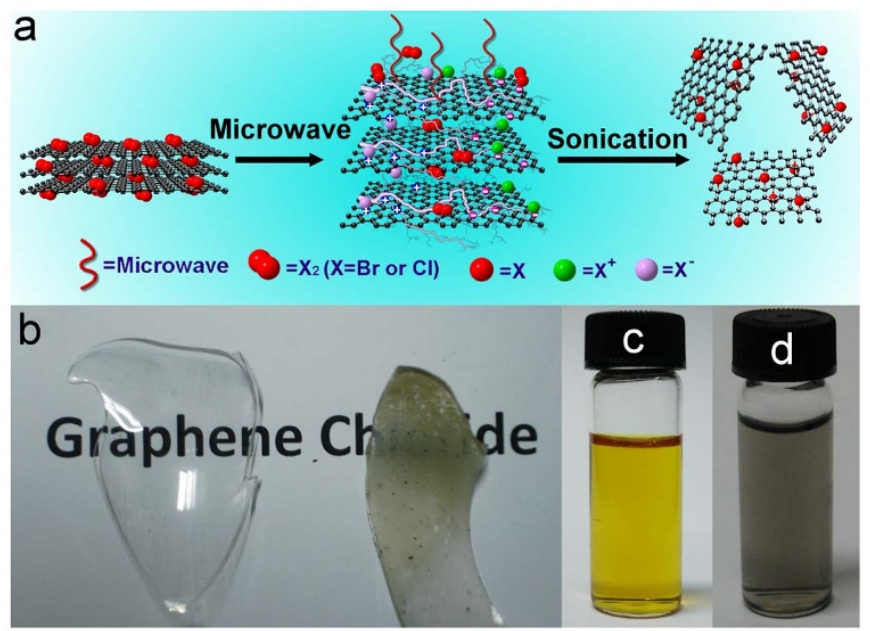

Figure $1 \mid$ (a) Schematic illustration of the chlorination and bromination processes. The halogen was first intercalated between the graphite layers. With the assistance of MiW-S the halogen then reacted with the graphite directly. In the solution-phase sonication step, the graphite halide was exfoliated to single layer G-X. (b) Photographs of the graphite chloride coated on the inside surface of the flask. (c) Photograph of G-Cl dispersion in DMF. (d) Photograph of G-Br dispersion in DMF. ascribed to its lower degree of modification. Up on modification, $\mathrm{p}$ electron concentration and structural ordering decreased, accompanied with absorption decrease. Because modification degree of $\mathrm{G}-\mathrm{Cl}$ was much higher than $\mathrm{G}-\mathrm{Br}$, just similar with $\mathrm{GO}$, the $\mathrm{G}-\mathrm{Cl}$ dispersion was yellow while $\mathrm{G}-\mathrm{Br}$ dispersion was black. The accurate thicknesses of the G-X sheets were determined by atomic force microscopy (AFM). After measured one hundred flakes, the average topographic heights of $\mathrm{G}-\mathrm{Cl}$ and $\mathrm{G}-\mathrm{Br}$ were around $1.2 \mathrm{~nm}$ and $1.5 \mathrm{~nm}$, respectively (Figure $2 \mathrm{a}$ and $2 \mathrm{~b}$ ). These values are larger than the thickness of a typical pristine monolayer of graphene $(\sim 0.8 \mathrm{~nm})$, and are indicative of a large amount of $\mathrm{Cl} / \mathrm{Br}$ atoms attached on the surface of the graphene layer. AFM measurements also indicated that the most of the $\mathrm{G}-\mathrm{Cl}$ sheets were monolayers while only $5 \%$ of $\mathrm{G}-\mathrm{Br}$ sheets were monolayers. One hundred flakes were measured after annealing at $600^{\circ} \mathrm{C}$, the topographic heights of $\mathrm{G}-\mathrm{X}$ were reduced to $0.8 \sim 0.9 \mathrm{~nm}$ showing that most of the halogen atoms had been removed (Figure S2).

In order to characterize the morphology and crystal structure of the G-X materials, transmission electron microscopy (TEM) and selected area electron diffraction (SAED) were performed. TEM images (Figures $2 \mathrm{c}$ and $2 \mathrm{~d}$ ) show the membranes on a lacey carbon TEM grid. The G-X is transparent and exhibits a very stable nature under irradiation by the electron beam. The sheets are rippled and entangled with each other, similar to what is observed for monolayer graphene nanosheets. The SAED pattern of $\mathrm{G}-\mathrm{Cl}$ (the inset in Figure 2c) shows diffraction rings and less resolved diffraction dots, indicating that the crystallinity of the graphene sheet has been significantly diminished by incorporation of the chlorine atoms. The SAED pattern of G-Br (the inset in Figure 2d) shows well-defined diffraction spots in a hexagonal pattern, showing that the crystallinity of the $\mathrm{G}-\mathrm{Br}$ is much better than that of $\mathrm{G}-\mathrm{Cl}$, although still less than that of pristine graphene ${ }^{36}$.

\section{Discussion}

Because the halide groups in G-X should be capable of being replaced by a variety of organic functional groups, the proportion of halogen atoms covalently attached to graphene is very important in terms of

a
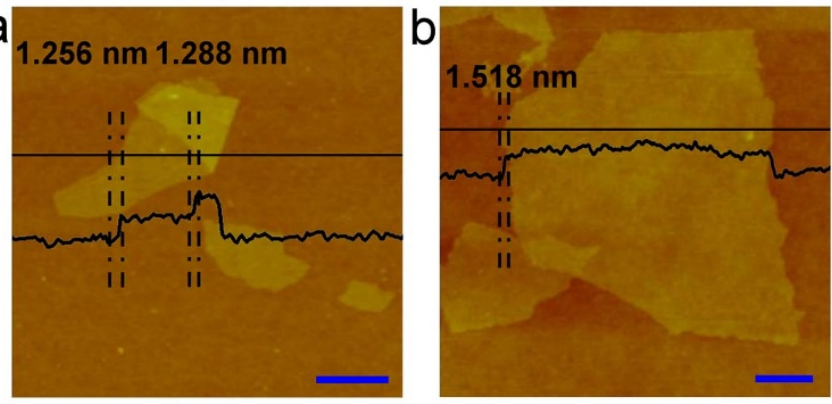

C
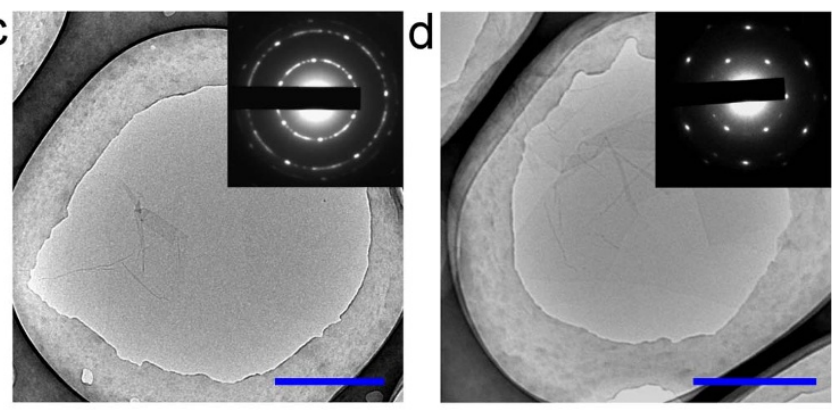

Figure 2 | AFM images of graphene sheets on a $\mathrm{SiO}_{2} / \mathrm{Si}$ substrate. (a) G-Cl, (b) G-Br, Bright-field TEM images of monolayer graphene and the corresponding electron diffraction patterns. (c) $\mathrm{G}-\mathrm{Cl}$, (d) G-Br. The blue bar is $500 \mathrm{~nm}$ in all images. 
further application of these materials. The halogen content was determined by X-ray photoelectron spectroscopy (XPS) and X-ray energy dispersive spectrometry (EDS) (Figures S3-S5). In the wide survey XPS spectra of $\mathrm{G}-\mathrm{Cl}$ and $\mathrm{G}-\mathrm{Br}$ (Figures $3 \mathrm{a}$ and $3 \mathrm{~b}$ ), the peaks at $284.8 \mathrm{eV}$ correspond to $\mathrm{C} 1 \mathrm{~s}$ of $\mathrm{sp}^{2}$ hybridized carbon. The obvious $\mathrm{Cl} 2 \mathrm{p}$ peaks located at $200.5 \mathrm{eV}\left(2 \mathrm{p}_{3 / 2}\right)$ and $201.8 \mathrm{eV}\left(2 \mathrm{p}_{1 / 2}\right)$ can be attributed to $\mathrm{C}-\mathrm{Cl}^{36}$, rather than likely possible contaminant species (e.g., peaks for $\mathrm{Cl}^{-}$would occur at $\sim 198.0 \mathrm{eV}$ and for $\mathrm{ClO}_{3}{ }^{-}$at $206 \mathrm{eV})^{36}$. The Br peak located at $70.8 \pm 0.2 \mathrm{eV}$ is also characteristic of $\mathrm{C}-\mathrm{Br}$, rather than possible contaminant species $\left(68.5 \mathrm{eV}\right.$ for $\mathrm{Br}^{-}$, $72.6 \mathrm{eV}$ and higher values for $\mathrm{BrO}_{3}{ }^{-}$, and $67.4 \pm 0.2 \mathrm{eV}$ for $\left.\mathrm{Br}_{2}\right)^{37,38}$. As a control, no $\mathrm{Cl}$ and $\mathrm{Br}$ peaks were detected in the expanded graphite (Figure S6). The XPS data therefore confirm that the $\mathrm{Cl}$ and $\mathrm{Br}$ atoms have been covalently functionalized on the graphene sheets (Figures 3c, 3d and S7). After considering the relevant atomic sensitivity factors, semi-quantitative analysis of the higher resolution data indicated a composition of 21 atom\% (ca. 44 weight $\%$ ) of $\mathrm{Cl}$ and 4 atom\% (ca. 21 weight\%) of $\mathrm{Br}$ in the respective samples ${ }^{38-41}$. G-Cl possesses a higher degree modification than $\mathrm{G}-\mathrm{Br}$ because liquid $\mathrm{Cl}_{2}$ is more reactive than $\mathrm{Br}_{2}$.
It has been reported that ultraviolet (UV) radiation can remove adsorbed gas molecules efficiently from reduced graphene oxide films or carbon nanotube films ${ }^{42,43}$. UV irradiation can therefore used to confirm that the materials contain covalently bonded halide groups rather than adsorbed $\mathrm{Cl}_{2}$ or $\mathrm{Br}_{2}$ molecules (Figures $3 \mathrm{e}$ and 3f). Both $\mathrm{G}-\mathrm{Cl}$ and a graphene film containing adsorbed $\mathrm{Cl}_{2}$ were illuminated by $254 \mathrm{~nm}$ UV radiation The $\mathrm{Cl}_{2}$ adsorbed in the graphene film was completely removed when the film was exposed to UV radiation for 20 minutes, while there was no noticeable change for the $\mathrm{G}-\mathrm{Cl}$ film. This provides clear evidence that the $\mathrm{Cl}$ atoms are covalently bonded to the graphene sheets. G-Br showed the same behavior. These observations confirm that the halogen atoms in G$\mathrm{Cl}$ and $\mathrm{G}-\mathrm{Br}$ are connected to the graphene sheets via covalent $\mathrm{C}-\mathrm{X}$ bonds. The presence of the characteristic $\mathrm{G}-\mathrm{Cl}$ and $\mathrm{G}-\mathrm{Br}$ stretching bands in the infrared (IR) spectra of the materials (Figure S8) provides additional confirmation of the covalent bonding in the $\mathrm{G}-\mathrm{X}$ materials.

Thermogravimetric analysis (TGA) provides still further evidence for the covalent attachment of halogen atoms to the graphene surface. Figure $3 \mathrm{~g}$ shows the TGA results of expanded graphite, $\mathrm{Cl}_{2} / \mathrm{Br}_{2}$
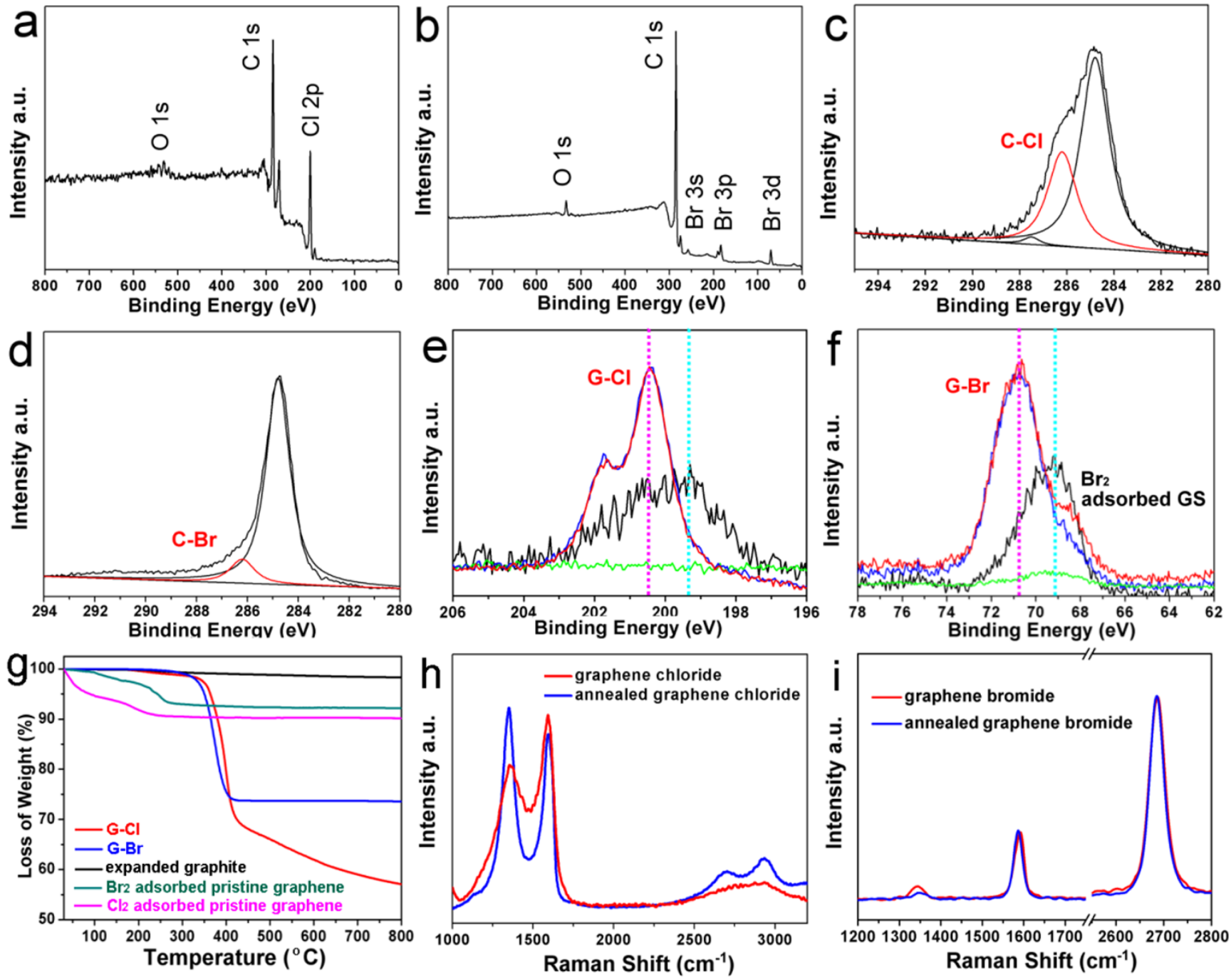

Figure 3 Wide survey XPS spectra of the graphene. (a) G-Cl, (b) G-Br. High-resolution XPS spectra of the graphene. (c) G-Cl, (d) G-Br. Deconvolution revealed the presence of C-C $(\sim 284.8 \mathrm{eV}), \mathrm{C}-\mathrm{O}$ and $\mathrm{C}-\mathrm{Br}(\sim 286.2 \mathrm{eV}), \mathrm{C}=\mathrm{O}(\sim 287.8 \mathrm{eV})$ and $\mathrm{C}-\mathrm{Cl}(\sim 286.2 \mathrm{eV})$ species in the films. The insets show high-resolution XPS spectra of the peaks. (e) $\mathrm{G}-\mathrm{Cl}$ (red), $\mathrm{G}-\mathrm{Cl}$ after being irradiated by $\mathrm{UV}$ (blue), $\mathrm{Cl}_{2}$ adsorbed on graphene (black), $\mathrm{Cl}_{2}$ adsorbed on graphene after being irradiated by UV (green). (f) G-Br (red), G-Br after being irradiated by UV (blue), $\mathrm{Br}_{2}$ adsorbed on graphene (black), $\mathrm{Br}_{2}$ adsorbed on graphene after being irradiated by UV (green). (g) TGA traces of the graphene samples. (h) Raman spectra of G-Cl. (i) Raman spectra of $\mathrm{G}-\mathrm{Br}$. 

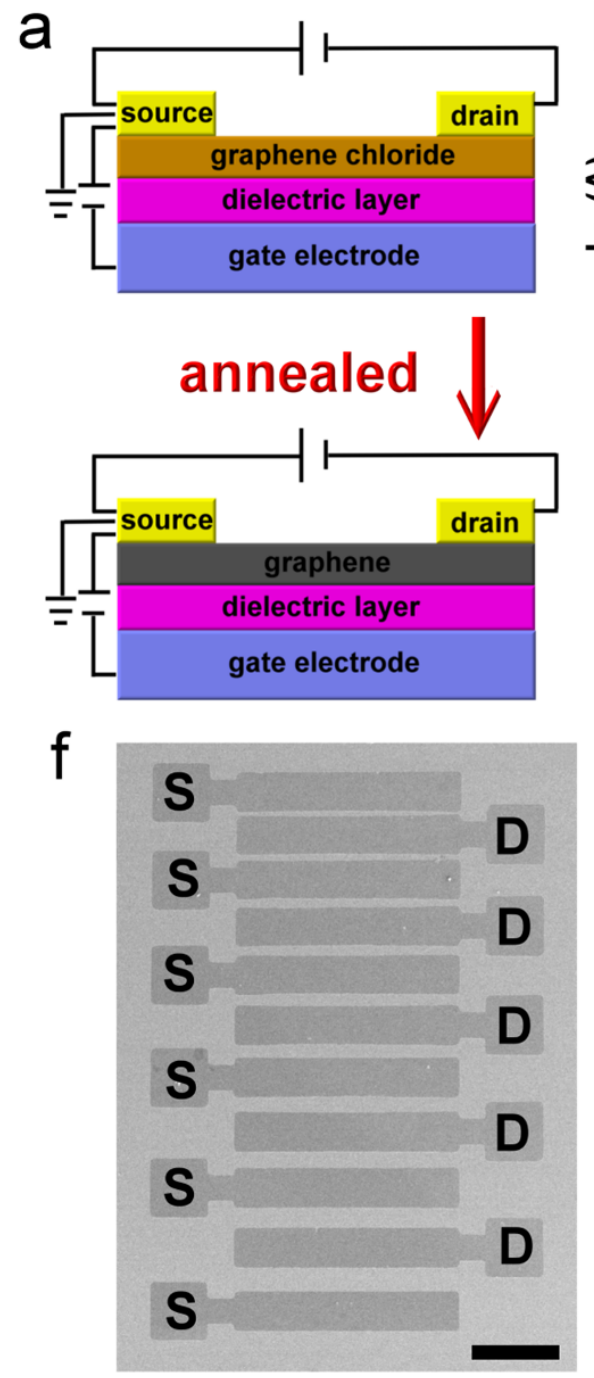
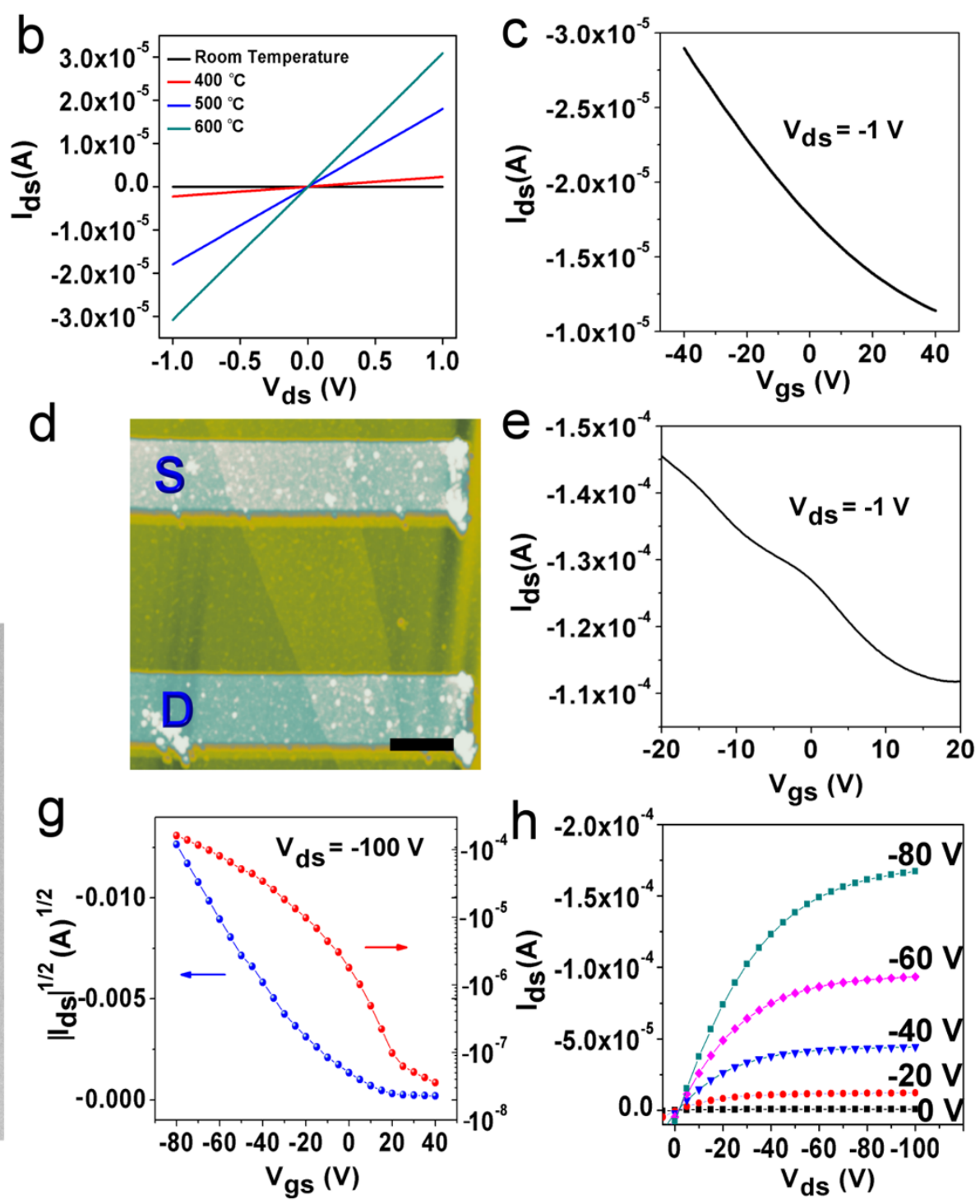

Figure $4 \mid$ (a) Schematic illustration of the fabrication of a G-Cl device. (b) Current-voltage $\left(I_{\mathrm{ds}}-V_{\mathrm{ds}}\right)$ characteristics of the same device annealed at different temperatures. (c) Current-gate voltage $\left(I_{\mathrm{ds}}-V_{\mathrm{gs}}\right)$ curves for a G-Cl device measured in air, after annealing at $600^{\circ} \mathrm{C}$. (d) AFM image of a G-Br FET device. (e) Current - gate voltage $\left(I_{\mathrm{ds}}-V_{\mathrm{gs}}\right)$ curves for the device in (d). (f) SEM image of annealed G-Cl electrode arrays with different widths (the black bar is $500 \mathrm{~nm})$. (g) Current - gate voltage $\left(I_{\mathrm{ds}}-V_{\mathrm{gs}}\right)$ curves for a pentacene device with a graphene electrode. (h) $I_{\mathrm{ds}}-V_{\mathrm{ds}}$ characteristics at various $V_{\mathrm{g}}$ for the device $(\mathrm{g})$ in air.

adsorbed on pristine graphene and G-X samples. Samples were heated from room temperature to $800^{\circ} \mathrm{C}$ under nitrogen and weight losses were monitored. The onset of decomposition occurred at temperatures $\left(\mathrm{T}_{\mathrm{d}}\right)$ of $366^{\circ} \mathrm{C}$ for $\mathrm{G}-\mathrm{Cl}$ and $344^{\circ} \mathrm{C}$ for $\mathrm{G}-\mathrm{Br}$ respectively (Figure $3 \mathrm{~g}$ and Figure S9), and can be attributed to the breaking of C$\mathrm{Cl} / \mathrm{C}-\mathrm{Br}$ bonds. Such high $\mathrm{T}_{\mathrm{d}}$ values and the abrupt losses in weight offer strong evidence that the $\mathrm{Cl} / \mathrm{Br}$ atoms are covalently bonded to the graphene, since the weight losses for the $\mathrm{Cl}_{2} / \mathrm{Br}_{2}$-adsorbed graphite samples occurred gradually from $\sim 50^{\circ} \mathrm{C}$ to $\sim 250^{\circ} \mathrm{C}$. In addition, after annealing at $600^{\circ} \mathrm{C}$, nearly all the $\mathrm{Cl}$ atoms were removed from $\mathrm{G}-\mathrm{Cl}$ since the $\mathrm{Cl} 2 \mathrm{p}$ peaks located at $200.5 \mathrm{eV}\left(2 \mathrm{p}_{3 / 2}\right)$ and $201.8 \mathrm{eV}$ $\left(2 \mathrm{p}_{1 / 2}\right)$ almost disappeared from the XPS (as shown in Figure S10).

The Raman spectra of G-X were recorded using the $514 \mathrm{~nm}$ excitation line (Figures $3 \mathrm{~h}$ and $3 \mathrm{i}$ ). The intensity of the $\mathrm{D}$ band in the Raman spectra of graphene materials is an indication of the level of defects. The obvious D peak located at $1359 \mathrm{~cm}^{-1}$ in the Raman spectrum of $\mathrm{G}-\mathrm{Cl}$ (Figure $3 \mathrm{~h}$ ) indicates that during the chlorination process, large quantities of covalent $\mathrm{C}-\mathrm{Cl}$ bonds were formed in graphene sheets, and thus a high degree of crystalline disorder was generated by the transformation from $\mathrm{sp}^{2}$ to $\mathrm{sp}^{3}$ configuration. The $\mathrm{D}$ peak $\left(\sim 1350 \mathrm{~cm}^{-1}\right)$ of $\mathrm{G}-\mathrm{Br}$ (Figure $3 \mathrm{i}$ ) is weaker than that of $\mathrm{G}-\mathrm{Cl}$, possibly due to the lower degree of modification. In the spectrum of $\mathrm{G}-\mathrm{Cl}$ the overtone of the $\mathrm{D}$ peak, denoted the $2 \mathrm{D}$ peak, nearly vanished, and this is also evidence of a high degree of structural disorder. Compared with the band for pristine graphene, the strong and symmetric $2 \mathrm{D}$ peak of $\mathrm{G}-\mathrm{Br}$ was blue-shifted $\left(8 \mathrm{~cm}^{-1}\right)$ and became broader, consistent with the small extent of modification by $\mathrm{Br}$. The decrease in chlorine content on heating (see above) shows that the structure of graphene chloride can be recovered by annealing at high temperature. Consistent with this, the Raman spectrum of annealed $\mathrm{G}-\mathrm{Cl}$ shows a higher $\mathrm{D} / \mathrm{G}$ intensity ratio than that for G$\mathrm{Cl}$, which is indicative of the creation of numerous new graphitic domains that are smaller in size than the ones present in exfoliated $\mathrm{G}-\mathrm{Cl}$. The 2D peak in the Raman spectrum of thermally annealed G$\mathrm{Cl}$ is more obvious than the peak in the spectrum of $\mathrm{G}-\mathrm{Cl}$, which is also evidence for the increased crystallinity after thermal annealing.

The structure recovery was accompanied by an improvement in electronic properties. G-Cl-based field-effect transistors (FETs) were 
fabricated with a top-contact configuration. A thin film of about 1$3 \mathrm{~nm}$ (1-3 layers) was spin-coated on a $\mathrm{Si} / \mathrm{SiO}_{2}$ substrate at room temperature (Figure $4 \mathrm{a}$ ). An n-type $\mathrm{Si}$ wafer with a $\mathrm{SiO}_{2}$ layer of $300 \mathrm{~nm}$ was used as the gate, and gold source and drain contacts $(30 \mathrm{~nm})$ were deposited on the $\mathrm{G}-\mathrm{Cl}$ film through a shadow mask. Figure $4 \mathrm{~b}$ shows that the conductivity of the $\mathrm{G}-\mathrm{Cl}$ film increased significantly after annealing for $15 \mathrm{~min}$ at different temperatures in vacuum. The pristine $\mathrm{G}-\mathrm{Cl}$ thin film was an insulator while after annealing at $600^{\circ} \mathrm{C}$, the conductivity of the $\mathrm{G}-\mathrm{Cl}$ thin film improved dramatically (Figure 4b). Devices fabricated using a thermally annealed $\mathrm{G}-\mathrm{Cl}$ thin film show p-type behavior in air (Figure $4 \mathrm{c}$ and S11). Due to the limited yield of G-Br samples, electron-beam lithography was used to fabricate G-Br FETs (Figure 4d). Figure 4e shows a typical current-voltage $\left(\mathrm{I}-\mathrm{V}_{\mathrm{g}}\right.$ ) curve of a single layer $\mathrm{G}-\mathrm{Br}$ measured under ambient conditions (source-drain bias voltage $-1 \mathrm{~V}$ ). The average two-dimensional resistivity (defined as RW/L, where $\mathrm{R}$ is the resistance of the device and $\mathrm{W}$ and $\mathrm{L}$ are the width and channel length, respectively, of the graphene sheets) of the graphene sheets was less than $10 \mathrm{k} \Omega$, which is close to the value for pristine graphene and suggests the graphene sheets have good quality ${ }^{44,45}$. In case of the modified graphene, foreign atoms and topological defects (as indicated by the TEM images and Raman spectra discussed above) are introduced into the graphene lattice, causing the observed decrease in the conductivity.

When incorporated as the electrode in electronic devices, graphene offers the advantages of low resistivity, high chemical stability, and mechanical strength. The good solubility of $\mathrm{G}-\mathrm{Cl}$ and its low cost as a precursor for the fabrication of graphene electrodes fulfill the requirements for the realization of large-area and economical organic electronic devices such as organic FETs, organic photovoltaics or organic light-emitting diodes. We patterned G-Cl $45 \mathrm{~nm}$ thin film electrode arrays by a solution-processed plasma etching approach (Figures $4 \mathrm{f}$ and S12) ${ }^{46}$. After annealing at $600^{\circ} \mathrm{C}$, the resulting graphene layer was deposited on the electrodes. The device characteristics of a pentacene FET with the graphene electrode in bottom contact geometry showed a mobility of $0.35 \mathrm{~cm}^{2} \mathrm{~V}^{-1} \mathrm{~s}^{-1}$ (Figures $4 \mathrm{~g}$ and $4 \mathrm{~h}$ ), which is higher than the corresponding values for an FET with annealed GO electrodes and bottom contact $\mathrm{Au}$ electrodes $\left(0.2 \mathrm{~cm}^{2} \mathrm{~V}^{-1} \mathrm{~s}^{-1}\right.$ and $0.06 \mathrm{~cm}^{2} \mathrm{~V}^{-1} \mathrm{~s}^{-1}$, respectively). The higher mobility of the former FET can ascribed to be suitable work function of graphene (4.7-4.9 eV), which enables ohmic hole injection into organic semiconductors with comparable HOMO energy levels, as well as the excellent graphene/pentacene contact ${ }^{9}$. Therefore, when an organic solvent process is required, $\mathrm{G}-\mathrm{Cl}$ is undoubtedly more attractive than GO as a graphene precursor.

It is well-known that organic chlorides are one of the most important organic precursors in many organic chemical reactions, such as substitution and metal-catalyzed cross coupling reactions. Therefore G-X should also be an important versatile precursor and intermediate material, which could be further modified with a variety of organic groups, in order to introduce new functionality or to fabricate new composite materials. For example, the halogen atoms in $\mathrm{G}-\mathrm{X}$ could be substituted by laurylamine under conventional organic reaction conditions (Figure 5a). The substitution can be confirmed by XPS (Supporting Information, Figures S13 and S14). The Cl 2p peaks located at $200.5 \mathrm{eV}\left(2 \mathrm{p}_{3 / 2}\right)$ and $201.8 \mathrm{eV}\left(2 \mathrm{p}_{1 / 2}\right)$ diminished after the reaction. $\mathrm{G}-\mathrm{Br}$ was more reactive than $\mathrm{G}-\mathrm{Cl}$, and the halide groups could be nearly completely substituted by laurylamine. The $\mathrm{Br} 3 \mathrm{~d}$ peak located at $70.8 \mathrm{eV}$ in the XPS of G-Br disappeared after the reaction. A new peak located at $400.9 \mathrm{eV}$ corresponding to $\mathrm{N} 1 \mathrm{~s}$ appeared. With mild sonication, the product could be redispersed in DMF forming a homogeneous $\mathrm{G}-\mathrm{N}$ suspension. The accurate thicknesses of single sheets deposited on the $\mathrm{Si} / \mathrm{SiO}_{2}$ substrate were characterized by AFM (Figures $5 \mathrm{~b}$ and $5 \mathrm{c}$ ). Due to the long aliphatic chains, the thickness of remodified graphene sheets was around $2.6 \mathrm{~nm}$, much thicker than for $\mathrm{G}-\mathrm{X}$.

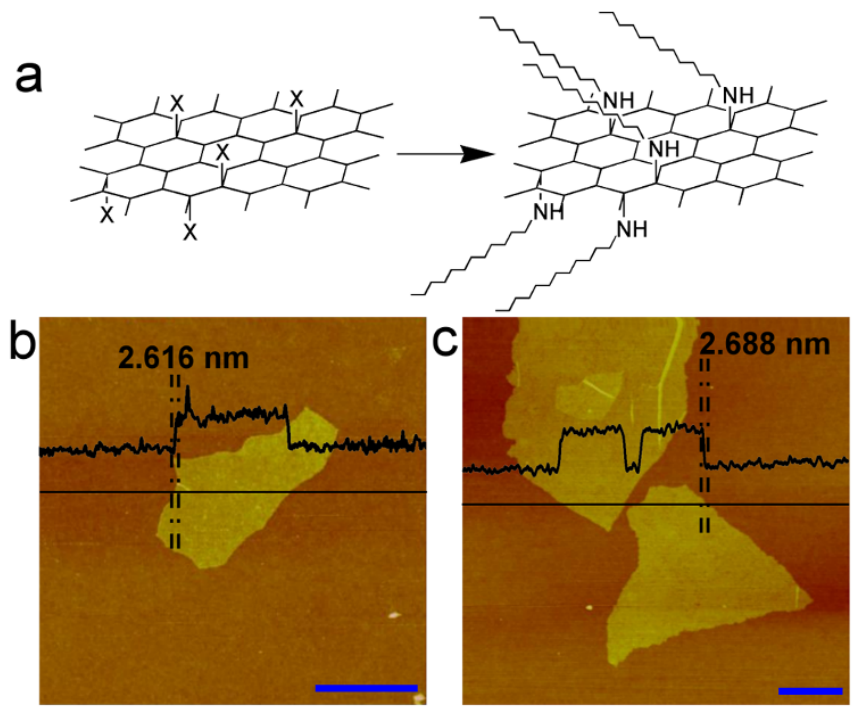

Figure 5 | (a) Schematic illustration of the substitution reaction of G-X. AFM images of G-NHC ${ }_{12} \mathrm{H}_{25}$ formed by (b) reaction of $\mathrm{G}-\mathrm{Cl}$, and (c) reaction of $\mathrm{G}-\mathrm{Br}$.

In conclusion, we have synthesized graphite chloride and bromide with a high degree of halogen incorporation for the first time, with the assistance of microwave sparks. The modified graphite could be directly exfoliated into single layer graphene halide by sonication. The structure and electronic properties of graphene could be recovered after thermal annealing of the graphene halide. OFETs based on these solution-processed $\mathrm{G}-\mathrm{Cl}$ electrodes arrays show high mobilities, even better than those using GO or gold electrodes. More importantly, the graphene halide could be further modified by other organic functional groups. The chlorinated and brominated graphene materials thus offer a novel pathway for the synthesis of a wide spectrum of graphene derivatives suitable for numerous potential applications.

\section{Methods}

Materials. Acid-intercalated expandable graphite (particle size: $500 \mu \mathrm{m}$, the graphite had been intercalated with sulfuric acid and nitric acid) was kindly provided by Tianhe Inc. $\mathrm{Cl}_{2}$ (99.99\%) was purchased from Arkonic Gases \& Chemicals Inc. Other chemicals were purchased from Aldrich. The $\mathrm{Br}_{2}$ was distilled after treatment with $\mathrm{H}_{2} \mathrm{SO}_{4}$. The other reagents were used as received unless otherwise stated.

Chlorination of graphite. Expandable graphite $(100 \mathrm{mg})$ was sealed in a vacuum glass flask. The flask was equiped with a condenser pipe through which was circulated ethanol at $-60^{\circ} \mathrm{C}$. Then $20 \mathrm{~mL}$ of liquid $\mathrm{Cl}_{2}$ was added into the vaccum flask. The vial was irradiated in a microwave oven (Galanz, $700 \mathrm{~W}, 2450 \mathrm{MHz}$ ) for $5 \mathrm{~s}$ (whilst being cooled by liquid nitrogen). After reaction, the $\mathrm{Cl}_{2}$ was evaporated under vacuum. A dark yellow solid was coated on the inside surface of the flask. Then the sample was sonicated in a solution of $\mathrm{NaHSO}_{3}(\sim 1 \mathrm{M}$ in DMF), pure water, DMF and tetrahydrofuran. Finally the sample was annealed at $150^{\circ} \mathrm{C}$ under a vacuum of $\sim 10^{-3}$ mbar to afford $10 \mathrm{mg}$ of chlorinated graphite.

Bromination of graphite. Expandable graphite $(100 \mathrm{mg})$ was sealed in a glass flask and purged with high purity nitrogen for $2 \mathrm{~h}$. Then $20 \mathrm{~mL}$ of $\mathrm{Br}_{2}$ was added. After $1 \mathrm{~h}$, the flask was irradiated in a microwave oven (Galanz, $700 \mathrm{~W}, 2450 \mathrm{MHz}$ ) for $60 \mathrm{~s}$ (whilst being cooled by liquid nitrogen). The irradiation process was repeated five times. After the reaction, the $\mathrm{Br}_{2}$ was evaporated under vacuum. Then the sample was sonicated in a solution of $\mathrm{NaHSO}_{3}(\sim 1 \mathrm{M}$ in DMF), pure water, DMF and tetrahydrofuran. Finally, the sample was annealed at $150^{\circ} \mathrm{C}$ and under a pressure of $\sim 10^{-3}$ mbar to yield $90 \mathrm{mg}$ of brominated graphite.

G-Cl. A mixture of $30 \mathrm{~mL}$ of DMF and $1 \mathrm{mg}$ of graphite chloride was sonicated in a low power sonic bath for $1 \mathrm{~min}$ to form a homogeneous suspension. The suspension was centrifuged at $2000 \mathrm{rpm}$ for $15 \mathrm{~min}$ to separate the non-soluble material from the solution. A suspension with predominantly single layer graphene sheets retained in the supernatant was obtained.

G-Br. A mixture of $30 \mathrm{~mL}$ of DMF and $1 \mathrm{mg}$ of graphite bromide was sonicated in a low power sonic bath for $30 \mathrm{~min}$ to form a homogeneous suspension. The suspension 
was centrifuged at $2000 \mathrm{rpm}$ for $15 \mathrm{~min}$ to separate the non-soluble material from the solution. A suspension with single layer graphene sheets retained in the supernatant was obtained.

G-N. A well dispersed suspension of $\mathrm{G}-\mathrm{Br}$ in DMF $(100 \mathrm{~mL})$ was refluxed in the presence of $0.5 \mathrm{~g}$ of laurylamine and $0.2 \mathrm{~g}$ of $\mathrm{K}_{2} \mathrm{CO}_{3}$, at $80^{\circ} \mathrm{C}$ for $3 \mathrm{~h}$, in a $\mathrm{N}_{2}$ atmosphere. At the end of the reaction, the suspension was filtered through a $0.4 \mu \mathrm{m}$ polyvinylidene fluoride (PVDF) membrane, and then the product was washed with $250 \mathrm{~mL}$ of water and $200 \mathrm{~mL}$ of chloroform. The sample was dried in a quartz tube at $120^{\circ} \mathrm{C}$ under a vacuum of $\sim 10^{-3} \mathrm{mbar}$.

Characterization. The samples were characterized using SEM (Hitachi S -4800 , $15 \mathrm{kV}$ ), X-ray EDS (fitted to the SEM), AFM (Multimode Nanoscope V), TEM (JEM-2010, $200 \mathrm{kV}$ ), SAED (fitted to the TEM), Raman spectrometer (Lab Ram HR800, with laser excitation at $514 \mathrm{~nm}$ ), XPS (ESCA Lab220I-XL) and TGA (EXSTAR 6000 TG/DTA 6300), and infrared thermometer (Raytek Raynger 3i).

1. Novoselov, K. S. et al. Electric field effect in atomically thin carbon films. Science 306, 666-669 (2004).

2. Novoselov, K. S. et al. Two-dimensional atomic crystals. Proc. Natl. Acad. Sci. USA 102, 10451-10453 (2005).

3. Park, S. \& Ruoff, R. S. Chemical methods for the production of graphenes. Nat. Nanotechnol. 4, 217-224 (2009).

4. Allen, M. J., Tung, V. C. \& Kaner, R. B. Honeycomb carbon: A review of graphene. Chem. Rev. 110, 132-145 (2010).

5. Stoller, M. D., Park, S., Zhu, Y., An, J. \& Ruoff, R. S. Graphene-based ultracapacitors. Nano Lett. 8, 3498-3502 (2008).

6. Dikin, D. A. et al. Preparation and characterization of graphene oxide paper Nature 448, 457-460 (2007).

7. Stankovich, S. et al. Graphene-based composite materials. Nature 442, 282-286 (2006).

8. Ramanathan, T. et al. Functionalized graphene sheets for polymer nanocomposites. Nat. Nanotechnol. 3, 327-331 (2008).

9. Di, C. et al. Patterned graphene as source/drain electrodes for bottom-contact organic field-effect transistors. Adv. Mater. 20, 3289-3293 (2008).

10. Bunch, J. S. et al. Electromechanical resonators from graphene sheets. Science 315 490-493 (2007).

11. Rao, C. N. R., Sood, A. K., Subrahmanyam, K. S. \& Govindaraj, A. Graphene: The new two-dimensional nanomaterial. Angew. Chem. Int. Ed. 48, 7752-7777 (2009).

12. Chen, D., Tang, L. \& Li, J. Graphene-based material in electrochemistry. Chem. Soc. Rev. 39, 3157-3180 (2010).

13. Loh, K. P., Bao, Q., Ang, P. K. \& Yang, J. The chemistry of graphene. J. Mater. Chem. 20, 2277-2289 (2010)

14. Wu, J., Pisula, W. \& Müllen, K. Graphene as potential material for electronics. Chem. Rev. 107, 718-747 (2010).

15. Lu, X. \& Chen, Z. Curved $\pi$-conjugation, aromaticity, and the related chemistry of small fullerenes $\left(<\mathrm{C}_{60}\right)$ and single-walled carbon nanotubes. Chem. Rev. 105, 3643-3696 (2005).

16. Farmer, D. B., Lin, Y. M., Afzali-Ardakani, A. \& Avouris, P. Behavior of a chemically doped graphene junction. Appl. Phys. Lett. 94, 213106 (2009).

17. Englert, J. M. et al. Covalent bulk functionalization of graphene. Nat. Chem. $\mathbf{3}$, 279-286 (2011).

18. Brodie, B. C. Sur le poids atomique du graphite. Ann. Chim. Phys. 59, 466 (1860).

19. Staudenmaier, L. Verfahren zur Darstellung der Graphitsaure. Ber. Deut. Chem. Ges. 31, 1481 (1898).

20. Hummers, W. S. \& Offeman, R. E. Preparation of graphitic oxide. J. Am. Chem. Soc. 80, 1339 (1958).

21. Kuriakose, A. K. \& Margrave, J. L. Kinetics of reaction of elemental fluorine .4. Fluorination of graphite. J. Phys. Chem. 2772-2775 (1965).

22. Worsley, K. A. et al. Soluble graphene derived from graphite fluoride. Chem. Phys. Lett. 445, 51-56 (2007)

23. Li, X. et al. Highly conducting graphene sheets and Langmuir-Blodgett films. Nat. Nanotechnol. 3, 538-542 (2008).

24. Subrahmanyam, K. S., Vivekchand, S. R. C., Govindaraj, A. \& Rao, C. N. R. A study of graphenes prepared by different methods: Characterization, properties and solubilization. J. Mater. Chem. 18, 1517-1523 (2008).

25. Eda, G. \& Chhowalla, M. Chemically derived graphene oxide: Towards large-area thin-film electronics and optoelectronics. Adv. Mater. 22, 2393-2415 (2010).

26. Dreyer, D. R., Park, S., Bielawski, C. W. \& Ruoff, R. S. The chemistry of graphene oxide. Chem. Soc. Rev. 39, 228-240 (2010).

27. Li, B. et al. Photochemical chlorination of graphene. ACS Nano 5, 5957-5961 (2011)
28. Wu, J. et al. Controlled chlorine plasma reaction for noninvasive graphene doping. J. Am. Chem. Soc. 133, 19668-19671 (2011).

29. Friedrich, J. F. et al. Plasma-chemical bromination of graphitic materials and its use for subsequent functionalization and grafting of organic molecules. Carbon 48, 3884-3894 (2010)

30. Li, J., Vaisman, L., Marom, G. \& Kim, J. Br treated graphite nanoplatelets for improved electrical conductivity of polymer composites. Carbon 45, 744-750 (2007).

31. Schlögl, R. Modification of the electronic structure of graphite by intercalation, chlorination and ion etching. Surf. Sci. 189/190, 861-872 (1987).

32. Zheng, J. et al. Production of high-quality carbon nanoscrolls with microwave sparks assistance in liquid nitrogen. Adv. Mater. 23, 2460-2463 (2011).

33. Zheng, J. et al. Production of graphene nanospheres by annealing of graphene oxide in solution. Nano Res. 4, 705-711 (2011).

34. Lou, Z., Lu, Y., Somers, L. A. \& Johnson, A. T. C. High yield preparation of macroscopic graphene oxide membranes. J. Am. Chem. Soc. 131, 898-890 (2009)

35. K Bethke, G. W. \& Ruess, A. D. Microwave-induced plasma shield propagation in rare gases. Phys. Fluids. 12, 822-835 (1969).

36. Ferrari, A. C. et al. Raman spectrum of graphene and graphene layers. Phys. Rev. Lett. 97, 187401-4 (2006).

37. Wagner, C. D., Riggs, W. M., Davis, L. E., Moulder, J. F. \& Muilenberg, G. E. Handbook of X-ray Photoelectron Spectroscopy: A Reference Book of Standard Data For Use In X-Ray Photoelectron Spectroscopy. 58-59 (Perkin-Elmer Corporation, 1979)

38. Papirer, E., Lacroix, R., Donnet, J. B., Nanse, G. \& Fioux, P. XPS study of the halogenation of carbon black-Part 1. Bromination. Carbon 32, 1341-1358 (1994).

39. Becerril, H. A. et al. Evaluation of solution-processed reduced graphene oxide films as transparent conductors. ACS Nano. 2, 463-470 (2008).

40. Unger, E., Graham, A., Kreupl, F., Liebau, M. \& Hoenlein, W. Electrochemical functionalization of multi-walled carbon nanotubes for solvation and purification. Curr. Appl. Phys. 2, 107-111 (2002).

41. Widenkvist, E. et al. Mild sonochemical exfoliation of bromine-intercalated graphite: A new route towards graphene. J. Phys. D: Appl. Phys. 42, 112003 (2009).

42. Dua, V. et al. All-organic vapor sensor using inkjet-printed reduced graphene oxide. Angew. Chem. Int. Ed. 49, 2154-2157 (2010).

43. Li, J. et al. Carbon nanotube sensors for gas and organic vapor detection. Nano Lett. 3, 929-933 (2003).

44. Dan, Y., Lu, Y., Kybert, N. J., Luo, Z. \& Johnson, A. T. C. Intrinsic response of graphene vapor sensors. Nano Lett. 9, 1472-1475 (2009).

45. Reina, A. et al. Large area, few-layer graphene films on arbitrary substrates by chemical vapor deposition. NanoLett. 9, 30-35 (2009).

46. Pang, S., Tsao, H. N., Feng, X. \& Müllen, K. Patterned graphene electrodes from solution-processed graphite oxide films for organic field-effect transistors. Adv. Mater. 21, 3488-3491 (2009).

\section{Acknowledgements}

We acknowledge financial support from the National Natural Science Foundation of China (60911130231, 61171054, 20825208, 20973184, 61101051), the Major State Basic Research Development Program (2011CB932701, 2011CB932303, 2009CB623603, 2011CB808403), and the Chinese Academy of Sciences.

\section{Author contributions}

J.Z. designed and performed the experiments, analyzed data and wrote the paper, H.T.L., B.W. and C.A.D. analyzed data and wrote the paper, Y.L.G., T.W. and Y.G. analyzed data and discussed the results, Y.Q.L. and D.B.Z. discussed the results and implications, commented on the manuscript at all stages, and Y.Q.L. revised and finalized the manuscript

\section{Additional information}

Supplementary information accompanies this paper at http://www.nature.com/ scientificreports

Competing financial interests: The authors declare no competing financial interests.

License: This work is licensed under a Creative Common

Attribution-NonCommercial-NoDerivative Works 3.0 Unported License. To view a copy of this license, visit http://creativecommons.org/licenses/by-nc-nd/3.0/

How to cite this article: Zheng, J. et al. Production of Graphite Chloride and Bromide Using Microwave Sparks. Sci. Rep. 2, 662; DOI:10.1038/srep00662 (2012). 